\title{
Sieve Method: Sieve the Forward and Reverse In One Time
}

\author{
Sai Chuen Hui \\ Frontier Science Research Institute of Hong Kong Limited
}

\begin{abstract}
This article carries on " Sieve Method: Sieve the Forward and Reverse In One Time" through establishing the dual element set and forms a new foundamental mathematical theory to sieve odd prime number pairs. The conclusion verifies the formula that calculating the total number of pairs that any even number is the sum of two odd prime numbers, and the minimum is that when an even number is extacted of root, the total number of odd prime number pairs is never less than one, so the Goldbach Conjecture is solved.
\end{abstract}

Keywords-sieve Method; sieve the forward and reverse in one time; prime number pairs; dual element set

\section{Positive And NeGative ScREening}

The only assumption is that "prime" is the only element in the mathematics of mass energy (matter and energy).

Example: take a $\mathrm{W}=26$ even after prescribing the prime number of prime sieve to 2, 3, 5 (red):

Positive subsets: $+\mathrm{S}=01$ (2) (3) 4 (5) 6789101112 1314151617181920212223242526 (positive energy of superscript)

Reverse subsets: $-\mathrm{S}=262524232221201918171615$ 14131211109876 (5) 4 (3) (2) 10 (subscript negative energy)

(1) [theorem 1] Goldbach's conjecture for the exact solution of the formula:

$$
\begin{aligned}
& Z_{w}(w)=Z_{p . q}(w)+2 Z_{a}(w) \cdots(1.1)
\end{aligned}
$$

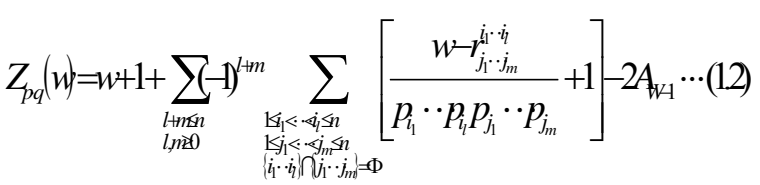

(2) the theorem 2 any even $\mathrm{W}$ table as the sum of two odd primes $\mathrm{p}+\mathrm{q}$ of odd prime number is at least $\mathrm{W}$ after prescribing an odd prime number, and a constant total of not less than 1 .

$$
\begin{aligned}
& Z_{w}^{\prime}(w) \geq\lfloor\pi(\sqrt{w})-1\rfloor+A_{w-1} \\
& A_{w-1}=\left\{\begin{array}{llll}
1 & w-1 & \text { prime number } & \left(\begin{array}{c}
1 \\
w-1
\end{array}\right) \text { Not sifted } \\
0 & w-1 & \text { Is } & \text { a } \\
& & \text { factor }\left(\begin{array}{c}
1 \\
w-1
\end{array}\right) \text { To be sifted }
\end{array}\right.
\end{aligned}
$$

Checking calculation: Taking the minimum value $=6$ of the even number, the limit value.

\section{Goldbach's conjecture}

[theorem 1-2] is confirmed by the appraisal document of the United States intellectual property rights bureau and the certificate of authorization of the 2012.4.4.

After 51 years (1962-2012) and 17 Chinese University professor... Hong Kong has Qiwen professor Su Dazhu, Ph.D., Southern China Normal University clock set column number of tutors, Xiamen University mathematician Li Wenqing guiding the audit results. Opening an equation to solve two unknowns is an alternative mathematics.

If the formula (1.1) is used to calculate the "heavy prime" table, "double key lock password" "DNA sequence" "atomic energy meter"... All the "universe tables" can be accurately looked up! The realization of Ai Bernsttein "... How to establish an aesthetic system that can be strictly expressed by a formula. " A mathematical description of the lifetime of the universe!

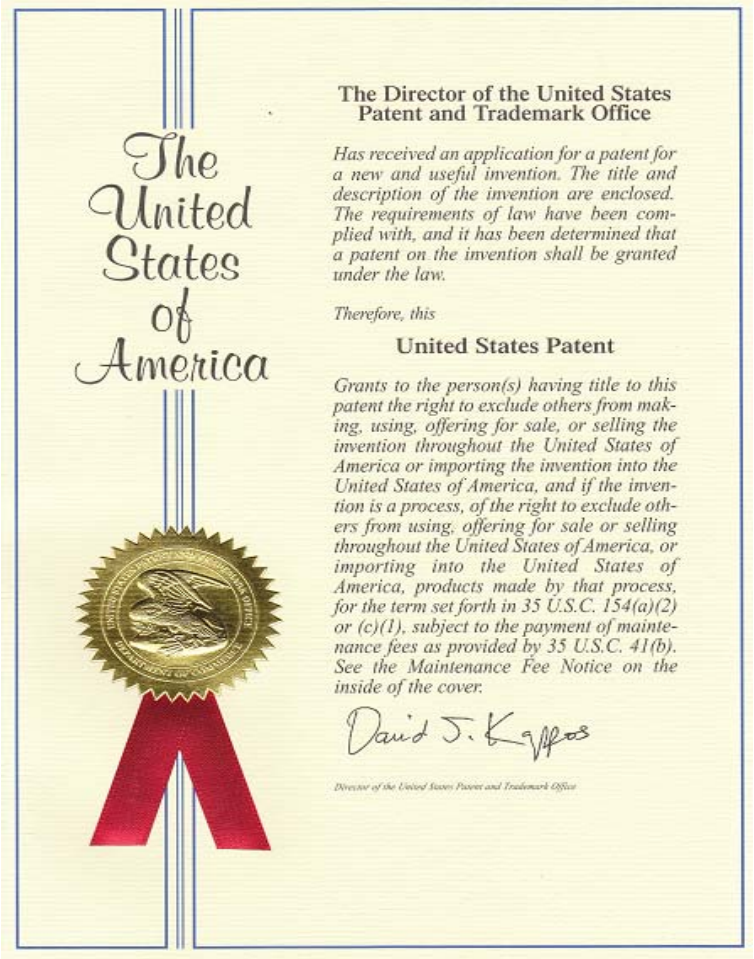

FIGURE I. US PATENT CERTIFICATE 


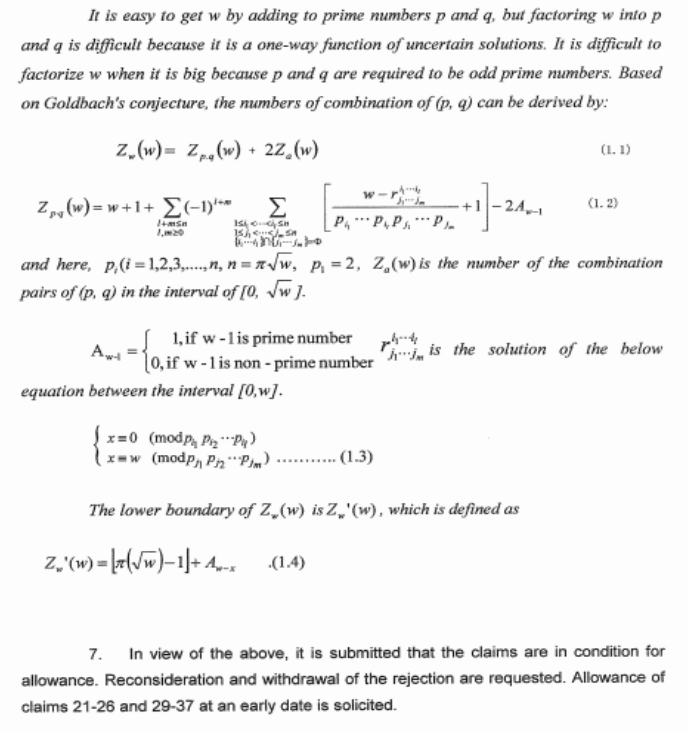

$\begin{array}{lllllllllllllll}\mathrm{S}^{+}= & 0 & 1 & 2 & 3 & 4 & 5 & 6 & 7 & 8 & 9 & 10 & 11 & 12 & 13 \\ \mathrm{~S}^{-}= & 26 & 25 & 24 & 23 & 22 & 21 & 20 & 19 & 18 & 17 & 16 & 15 & 14 & 13\end{array}$

By combining the two subsets, we can see that the sum of any arbitrary pair of numbers is equal $\mathbf{w}=\mathbf{2 6}$.

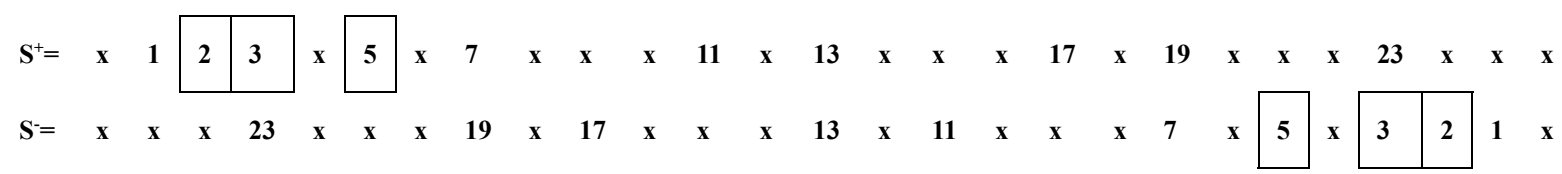

$\bigvee_{\mathbf{w}}=\sqrt{\mathbf{2 6}}=\mathbf{5 . 0 9 9}$, which after rounding, the prime factors are 2,3 and $\mathbf{5}$.

This gives us two pairs of odd primes of known elements: $3+23$ and $23+\mathbf{3}$, along with three pairs of odd primes with

\section{BACKGROUND}

In a letter to Euler on June $7^{\text {th }} 1742$, Goldbach proposed the conjecture expressed this in two forms:

(A) Every even integer $\geq 6$ can be written as the sum of two odd primes.

(B) Ever odd integer $\geq 9$ can be written as the sum of three odd primes.

We call (A) the strong conjecture and (B) the weak conjecture.

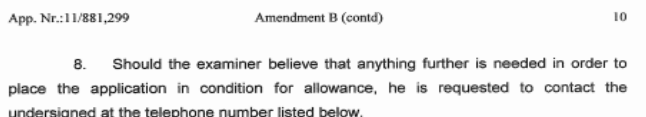

place the application in condition for allowance, he is requested to contact the

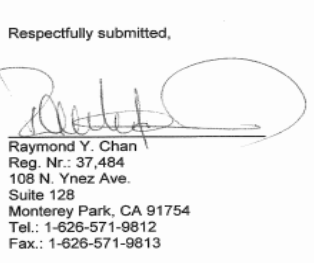

CERTIFICATE OF MAILING

1 hereby certify that this correspondence is being deposited with the United States
Postal Service with proper postage as first class mail in an envelope addressed to Postal Service with proper postage as first class mail in an envelope addressed to:
"Commissioner for Patents, P.O. Box 1450 . Alexandria, VA 22313-1450" or being

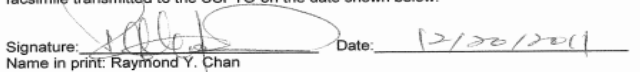

FIGURE II. IDENTIFICATION DOCUMENTS OF THE UNITED STATES INTELLECTUAL PROPERTY OFFICE

Take any even number, $\mathbf{w}=\mathbf{2 6}$ say.

We look at the set of its non-negative integers:

Forward Subset: $\mathrm{S}^{+}=\mathbf{0}, \mathbf{1}, \mathbf{2}, \ldots, 24,25,26$

Backward Subset: $S^{-}=\mathbf{2 6}, 25,24, \ldots, 2,1,0$ 


$$
A_{w-1}=\left\{\begin{array}{l}
1, \text { if } w-1 \text { is prime (i.e. }(1, w-1) \text { hasn't been sieved) } \\
0, \text { if } w-1 \text { is not a prime (i.e. }(1, w-1) \text { has been sieved) }
\end{array}\right.
$$

- Let $Z_{a}(w)$ be the number of pairs $\left(p_{i}, w-p_{i}\right)$ and hence Mr. Hui proposes that: where $p_{i}$ is a prime between $[0, \sqrt{w}]$ and $w-p_{i}$ is also prime.

$$
Z_{w}(w)=Z_{p q}(w)+2 Z_{a}(w)
$$

- Let $Z_{p q}(w)$ be the number of pairs $(p, q)$ that satisfies $w=p+q$ but falls outside the categories of with $Z_{a}(w)$ and $A_{w-1}$.

This covers all types of number pairs we will encounter,

$$
Z_{p q}(w)=w+1+\sum_{\substack{l+m \leq n \\ l, m \geq 0}}(-1)^{l+m} \sum_{\substack{1 \leq i_{1}<.<i_{l} \leq n \\ 1 \leq j_{1}<.<j_{m} \leq n \\\left\{i_{1} . . i_{l}\right\} \square\left\{j_{1} . . j_{m}\right\}=\phi}}\left[\frac{w-r_{j_{1} \ldots j_{m}}^{i_{1} \ldots i_{l}}}{p_{i_{1}} \ldots p_{i_{l}} p_{j_{1}} \ldots p_{j_{m}}}+1\right]-2 A_{w-1}
$$

where $r_{j_{1} \ldots j_{m}}^{i_{1} \ldots i_{l}}$ is the initial value of $S_{j_{1} \ldots j_{m}}^{i_{1} \ldots i_{l}}$, and $S_{j_{1} \ldots j_{m}}^{i_{1} \ldots i_{l}}=S^{p^{i_{1}}} \bigcap \cdots \bigcap S^{p^{i_{l}}} \bigcap S_{p_{j_{1}}} \bigcap \cdots \bigcap S_{p_{j_{m}}}$ with $S^{p_{i}}$ and $S_{p_{j}}$ being the resultant subsets that are obtained by sieving the top and bottom rows of $S$ by $p_{i}$ and $p_{j}$ respectively.

The lower bound of $Z_{w}(w)$, called $Z^{\prime}{ }_{w}(w)$, is:

$$
Z_{w}^{\prime}(w)=[\pi(\sqrt{w})-1]+A_{w-1}
$$

where $n=\pi(\sqrt{w})$ is the number of primes between $[0, \sqrt{w}]$.

It is easy to show that $[\pi(\sqrt{w})-1]+A_{w-1}$ is always greater than or equal to 1 (as there are infinitely many primes), and thus by combining (1) and (3), Goldbach's Conjecture can be verified. The author fully understands the implication of this statement and would like to clarify that the aims behind his theories are solely for the purpose of furthering any discussion in this field. This is because the basis of his proof is fundamentally based on his concept of a 2-Way Sieve, which he believes requires a much higher level of scrutiny than it currently receives. Otherwise, he is afraid that the concept would only be deemed worthy of ridicule by the worldwide community.

\section{PROOF}

Here is how the proof roughly goes:

Take the set $\mathrm{S}$

$$
\begin{gathered}
S=\left\{\left(\begin{array}{l}
0 \\
w
\end{array}\right),\left(\begin{array}{c}
1 \\
w-1
\end{array}\right),\left(\begin{array}{c}
2 \\
w-2
\end{array}\right), \ldots,\left(\begin{array}{c}
p_{i} \\
w-p_{i}
\end{array}\right), \ldots,\left(\begin{array}{c}
x \\
w-x
\end{array}\right), \ldots,\left(\begin{array}{c}
w-p_{i} \\
p_{i}
\end{array}\right), \ldots,\left(\begin{array}{c}
w-2 \\
2
\end{array}\right),\left(\begin{array}{c}
w-1 \\
1
\end{array}\right),\left(\begin{array}{l}
w \\
0
\end{array}\right)\right\} \\
\mathrm{B}(0) \quad \mathrm{C}(w-\sqrt{ } w)
\end{gathered}
$$

And divide by $\sqrt{w}_{\mathbf{w}}$ into three segments: $\mathrm{AB}, \mathrm{BC}$ and $\mathrm{CD}$. $\mathrm{AB}$ and $\mathrm{CD}$ are symmetrical. $p_{i}$ is a prime between $[0, \sqrt{w}]$ which we use to sieve the top and bottom rows of $S$ separately.

(a) Sieve the top row of $S$ (i.e. the Forward Sieve) 
using $p_{i}$, and call the resulting subset $S^{p_{i}}$ (note we will express $p_{i}$ as $p_{i_{1}} \ldots p_{i_{l}}, l \leq n$ ):

$$
\left.S^{p_{i}}=\left\{\left(\begin{array}{l}
0 \\
w
\end{array}\right),\left(\begin{array}{c}
p_{i} \\
w-p_{i}
\end{array}\right),\left(\begin{array}{c}
2 p_{i} \\
w-2 p_{i}
\end{array}\right) \ldots,\left(\begin{array}{c}
x \\
w-x
\end{array}\right), \ldots \ldots\right\}\right\} \quad x \equiv 0 \quad\left(\bmod p_{i}\right)
$$

The smallest $x$ could be is 0 , which we'll denote as $r^{p_{i}}=0$, the initial value of $S^{p_{i}}$. Remove $S^{p_{i}}$ from $S$ and note that $\left(\begin{array}{c}p_{i} \\ w-p_{i}\end{array}\right)$ is also sieved.

Backwards Sieve), expressing $p_{j}$ as $p_{j_{1}} \ldots p_{j_{m}}$, with $m \leq n$. We obtain the subset $S_{p_{j}}$ :

(b) Take $p_{j}$ and sieve the bottom row of $S$ (the

$$
S_{p_{j}}=\left\{\ldots \ldots,\left(\begin{array}{c}
w-x \\
x
\end{array}\right), \ldots,\left(\begin{array}{c}
w-2 p_{j} \\
2 p_{j}
\end{array}\right),\left(\begin{array}{c}
w-p_{j} \\
p_{j}
\end{array}\right),\left(\begin{array}{c}
w \\
0
\end{array}\right)\right\} \quad x \equiv w \quad\left(\bmod p_{j}\right)
$$

Since $w-x=\lambda p_{j}$ (with $\lambda$ an integer), we get $w-x \equiv 0 \quad\left(\bmod p_{j}\right)$, hence $x \equiv w\left(\bmod p_{j}\right)$. The minimum value of $\mathrm{x}$ is $r_{p_{j}}=\langle w\rangle_{p_{j}}\langle w\rangle_{p_{j} \text { being the }}$ remainder of w divided ${ }^{p_{j}}$. As before this is the initial value $S_{p_{j}}$, which we remove from $\mathrm{S}$ and noting again that $\left(\begin{array}{c}w-p_{j} \\ p_{j}\end{array}\right)$ is also sieved.

$\mathrm{S}$, all elements of the form $\left(\begin{array}{c}\lambda p_{i} \\ w-\lambda p_{i}\end{array}\right)$ are sieved from segments $\mathrm{AB}, \mathrm{BC}$ and $\mathrm{CD}$. This leaves us with pairs of odd primes $\left(\begin{array}{l}p \\ q\end{array}\right)$ in the BC, which we will denote as $Z_{p q}(w)$. Upon letting $2 Z_{a}(w)$ be the number of pairs $\left(\begin{array}{c}p_{i} \\ w-p_{i}\end{array}\right)$ between $\mathrm{AB}$ and $\mathrm{CD}$ where $p_{i}$ and $w-p_{i}$ are both prime, we get:

$$
S^{p^{i_{1}}}, S^{p^{i_{2}}}, S^{p^{i_{l}}} S_{p_{j_{1}}} S_{p_{j_{2}}, \ldots,} S_{p_{j_{m}} \text { from }}^{\text {subtracting }}
$$

$$
Z_{w}(w)=Z_{p q}(w)+2 Z_{a}(w)
$$

We begin by looking at the size of the sets:

$$
|S|=w+1,\left|S^{p_{i}}\right|=\left[\frac{w-r^{p_{i}}}{p_{i}}+1\right],\left|S_{p_{j}}\right|=\left[\frac{w-r_{p_{j}}}{p_{j}}+1\right]
$$

Suppose we know what $\pi(\sqrt{w})$ is. If we now consider the number of primes between $\mathrm{BD}$, we obtain the following:

$$
\pi(w)-\pi(\sqrt{w})=(w+1)-1-\sum_{i=1}^{n}\left[\frac{w-r^{p_{i}}}{p_{i}}+1\right]+\sum_{0 \leq I_{1}<I_{2} \leq N}^{n}\left[\frac{w-r^{p_{1} p_{2}}}{p_{1} \cdot p_{2}}+1\right]-\mathrm{L}+(-1)^{n}\left[\frac{w-r^{p_{1} \mathrm{~L} p_{N}}}{p_{1} \mathrm{~L} p_{N}}+1\right]
$$

Which simplifies to 


$$
\pi(w)-1=[\pi(\sqrt{w})-1]+w+\sum_{1 \leq l \leq n}(-1)^{l} \sum_{1 \leq i_{1}<.<i_{l} \leq n}\left[\frac{w-r^{i_{1} \cdots i_{l}}}{p_{i_{1}} \cdots p_{i_{l}}}\right]
$$

Now,

$$
\begin{aligned}
& Z_{p q}(w)=\left|S-\bigcup_{\substack{i=1 \\
j=1}}^{n} S_{p_{j}}^{p_{i}}\right|=|S|+\sum_{\substack{l+m \leq n \\
l, m \geq 0}}^{n}(-1)^{l+m} \sum_{\substack{1 \leq i_{1}<\mathrm{L}<i_{1} \leq n \\
1 \leq j_{1}<\mathrm{L}<j_{m} \leq n}}\left|S_{j_{1} \ldots j_{m}}^{i_{1} \ldots i_{l}}\right| \\
& =|S|+\sum_{\substack{0 \leq i \leq n \\
0 \leq j \leq n}}(-1)^{i+j} \sum_{\substack{1 \leq i_{i}<\mathrm{L}<i_{1} \leq n \\
1 \leq j_{1}<\mathrm{L}<j_{m} \leq n}}\left|S^{p_{i_{1}}} \mathrm{LS}^{p_{i_{l}}} S_{p_{j_{1}}} \mathrm{~L}_{p_{j_{m}}}\right| \\
& =|S|+\sum_{\substack{0 \leq i \leq n \\
0 \leq j \leq n}}\left|S_{p_{j}}^{p_{i}}\right|+\sum_{\substack{0 \leq i_{1}<i_{2} \leq n \\
0 \leq j_{1}<j_{2} \leq n}}\left|S^{p_{i_{1}}} S_{p_{j_{2}}}\right|-\sum_{\substack{0 \leq i_{i}<i_{i_{2}<i_{i} \leq n} \leq n \\
0 \leq j_{1}<j_{2}<j_{3} \leq n}}\left|S^{p_{i_{1}}} S_{p_{j_{2}}}^{p_{i_{2}}} S_{p_{j_{3}}}\right|+\mathrm{L}+(-1)^{l+m}\left|S^{p_{i_{1}}} \square S_{p_{j_{m}}}\right| \\
& =w+1-\sum_{\substack{0 \leq i \leq n \\
0 \leq j \leq n}}\left[\frac{w-r_{j}^{i}}{p_{i, j}}+1\right]+\sum_{\substack{0 \leq i_{1}<i_{2} \leq n \\
0 \leq j_{1}<j_{j} \leq n \\
\left\{i_{1} i_{2}\right\}\left\{j_{1} j_{2}\right\}}}\left[\frac{w-r_{j_{1} j_{2}}^{i_{1} i_{2}}}{p_{i} p_{j}}+1\right]-\sum_{\substack{0 \leq i_{1}<i_{2}<i 3 \leq n \\
0 \leq j_{1}<j_{2}<j 3 \leq n \\
\left\{i_{1}\left\llcorner i_{3}\right\}\left\{j_{1} \mathrm{~L} j_{3}\right\}\right.}}\left[\frac{w-r_{j_{1} \square j_{3}}^{i_{1} \square i_{3}}}{p_{i} \square p_{j}}+1\right] \\
& +\mathrm{L}+(-1)^{l+m}\left[\frac{w-r_{j_{1} L j_{m}}^{i_{1} i_{l}}}{p_{i} \mathrm{~L}_{j}}+1\right]-2 A_{w-1} \\
& =w+1+\sum_{\substack{l+m \leq n \\
l, m \geq 0}}(-1)^{l+m} \sum_{\substack{1<i_{1}<\ldots<i_{l} \leq n \\
1 \leq j_{1}<<j_{m} \leq n \\
\left\{i_{1} . i_{l}\right\} \leq\left\{j_{1} \ldots j_{m}\right\}=\phi}}\left[\frac{w-r_{j_{1} \ldots j_{m}}^{i_{1} \ldots j_{l}}}{p_{i_{1}} \ldots p_{i_{l}} p_{j_{1}} \ldots p_{j_{m}}}\right]-2 A_{w-1}
\end{aligned}
$$

Where

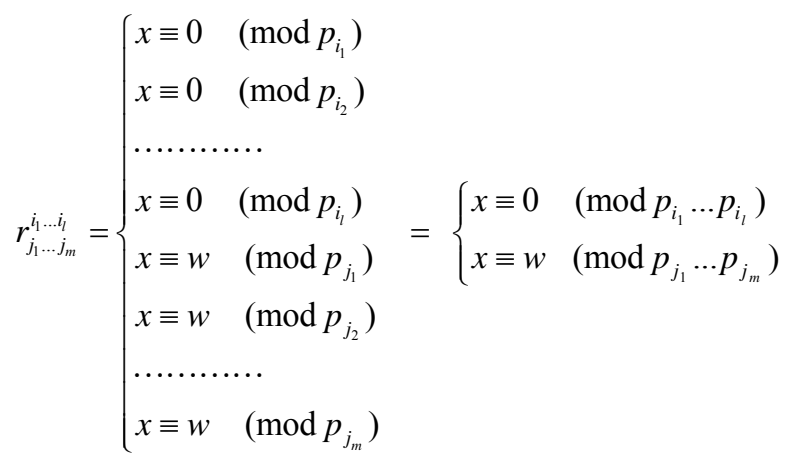

Moving on, we now show

$$
Z_{w}^{\prime}(w)=[\pi(\sqrt{w})-1]+A_{w-1}
$$


Prior to sieving, we can alter (2.1) slightly to express the sum of odd prime pairs $Z_{w}(w)$ and pairs that contain a

$$
2 Z_{w}(w)+Z_{p}(w)=2\left\{[\pi(\sqrt{w})-1]+w+\sum_{1 \leq l \leq n}(-1)^{l} \sum_{1 \leq i_{1}<.<i_{l} \leq n}\left[\frac{w-r^{i_{1} \cdots i_{l}}}{p_{i_{1}} \cdots p_{i_{l}}}+1\right]\right\}
$$

After applying the 2-Way Sieve, segments $\mathrm{AB}$ and $\mathrm{BD}$ are $\quad$ removed, leaving us with $Z_{p q}(w)$ in segment $\mathrm{BC}$, i.e.

$2 Z_{w}(w)+2 Z_{a}(w)=2\left\{[\pi(\sqrt{w})-1]+w+\sum_{1 \leq l \leq n}(-1)^{l} \sum_{1 \leq i_{1}<. \dot{\iota}_{l} \leq n}\left[\frac{w-r^{i_{1} \square i_{l}}}{p_{i_{1}} \square p_{i_{l}}}+1\right]\right\}-Z_{p}(w)-2[\pi(\sqrt{w})-1]-2 A_{w-1}$

And substituting in equation (1), we get:

$$
2 Z_{p q}(w)+Z_{p}(w)=2\left\{w+\sum_{1 \leq l \leq n}(-1)^{l} \sum_{1 \leq i_{1}<\ldots<i_{l} \leq n}\left[\frac{w-r^{i_{1} \cdots i_{l}}}{p_{i_{1}} \cdots p_{i_{l}}}+1\right]\right\}-2 A_{w-1}
$$

So equations (2.2) and (2.3) must be satisfied before and after sieving. Thus we can solve these simultaneously to get:

$$
\begin{aligned}
2 Z_{w}(w)-2 Z_{p q}(w) & =2[\pi(\sqrt{w})-1]+2 A_{w-1} \\
Z_{w}(w) & =[\pi(\sqrt{w})-1]+A_{w-1}+Z_{p q}(w)
\end{aligned}
$$

Now suppose that $Z_{p q}(w)=0$, then we are left with the minimum value of $Z_{w}(w)$, which is:

$$
Z^{\prime}{ }_{w}(w)=[\pi(\sqrt{w})-1]+A_{w-1}
$$

\section{SOME BRIEF EXAMPLES}

$w-1=10-1=9$ is not prime, so $A_{w-1}=0$.

1) Take $w=10$.

$$
n=\pi(\sqrt{10})=2, \quad p_{i_{1}}=p_{j_{1}}=2, \quad p_{i_{l}}=3, \quad p_{j_{m}}=3^{\prime}
$$

$$
\begin{aligned}
Z_{p q}(w) & =10+1-\left\{\left[\frac{10-0}{2}+1\right]+\left[\frac{10-1}{3}+1\right]+\left[\frac{10-1}{3^{\prime}}+1\right]\right\}+\left\{\left[\frac{10-4}{3.2}+1\right]+\left[\frac{10-4}{2.3^{\prime}}+1\right]\right\}-0 \\
& =11-(6+4+4)+(2+2)=11-14+4=1
\end{aligned}
$$

and $2 Z_{a}(w)=2$ (since we have $7+3$ and $3+7$ ).

$\therefore Z_{w}(w)=Z_{p q}(w)+2 Z_{a}(w)=1+2=3 \quad$ (the three pairs being $3+7,5+5$ and $7+3)$.

$$
Z^{\prime}{ }_{w}(w)=[\pi(\sqrt{w})-1]+A_{w-1}=2-1+0=1
$$

is also correct.

2) Take $w=12$

$$
w-1=12-1=11 \text { is prime, so } A_{w-1}=1 .
$$

$$
n=2 \quad p_{i_{l}}=2,3 \quad p_{j_{m}}=2^{\prime}, 3^{\prime} \quad r^{2.3}=r_{2^{\prime} 3^{\prime}}=\langle 12\rangle_{2^{\prime} \cdot 3^{\prime}}=0
$$
and $Z_{a}(w)=0$. 


$$
\begin{gathered}
Z_{p q}(w)=12+1-\left\{\left[\frac{12}{2}+1\right]+\left[\frac{12}{3}+1\right]\right\}+\left[\frac{12}{2.3}+1\right]-2 \\
=13-(7+5)+3-2=2 \\
\therefore Z_{w}(w)=Z_{p q}(w)+2 Z_{a}(w)=2+0=2 \\
\quad \text { (the two pairs being } 5+7 \text { and } 7+5)
\end{gathered}
$$

$$
Z^{\prime}{ }_{w}(w)=[\pi(\sqrt{w})-1]+A_{w-1}=2-1+1=2 \text { is }
$$
also correct.

Calculation :

$$
Z_{w}^{\prime}(w)=\lfloor\pi(\sqrt{w})-1\rfloor+A_{W+1}
$$

\section{REFERENCES}

[1] 2001-2004, the vice professor Song Fugao of Shen Zhen university calculated $w=6 \rightarrow 10^{7}$ comformed with (1.4)

[2] 2002,professor Zhu Lie of Su Zhou university calculated $W<20001$ comformed with (1.4)

[3] 2006,professor $\mathrm{Xu}$ Zuoming of Liao Ning university calculated $w=6 \rightarrow 10^{11}$ comformed with (1.4) 